\begin{tabular}{|c|c|}
\hline \multirow[t]{3}{*}{ Citation } & Xin Wang, Julian Stoev, Gregory Pinte, Jan Swevers (2012) \\
\hline & $\begin{array}{l}\text { Energy Optimal Point-to-Point Motion Using Model Predictive } \\
\text { Control }\end{array}$ \\
\hline & Dynamic Systems and Control Conference, 2012, pa e 267-273 \\
\hline Archived version & $\begin{array}{l}\text { Author manuscript: the content is identical to the content of the } \\
\text { published paper, but without the final typesetting by the publisher }\end{array}$ \\
\hline Published version & doi:10.1115/DSCC2012-MOVIC2012-8586 \\
\hline \multicolumn{2}{|l|}{ Journal homepage } \\
\hline \multirow[t]{2}{*}{ Author contact } & xin.wang@mech.kuleuven.be \\
\hline & phone number + $32(0) 16322222$ \\
\hline IR & https://lirias.kuleu en.be/handle/123456789/370251 \\
\hline
\end{tabular}




\section{ENERGY OPTIMAL POINT-TO-POINT MOTION USING MODEL PREDICTIVE CONTROL}

\author{
Xin Wang* \\ Jan Swevers \\ Department of Mechanical Engineering, \\ KU Leuven, Leuven, Belgium \\ Email: xin.wang@mech.kuleuven.be \\ Email: Jan.Swevers@mech.kuleuven.be
}

\author{
Julian Stoev \\ Gregory Pinte \\ Flanders' Mechatronics Technology Centre, \\ Leuven, Belgium \\ Email: Julian.Stoev@fmtc.be \\ Email: Gregory.Pinte@fmtc.be
}

\begin{abstract}
This paper discusses energy optimal point-to-point motion control for linear time-invariant (LTI) systems using energyoptimal Model Predictive Control (EOMPC). The developed EOMPC, which is based on time-optimal MPC, aims at performing energy-optimal point-to-point motions within a required motion time. Energy optimality is achieved by setting the object function of the EOMPC optimization problem equal to the system's energy losses. The key issue is to utilize the strategy of the prediction horizon to ensure that the motion time is exactly equal to the required motion time. Application of EOMPC on a badminton robot shows the practical applicability of the developed method. In addition, an experimental comparison with time-optimal MPC is provided.
\end{abstract}

\section{INTRODUCTION}

The interest in developing novel control approaches for minimizing the energy consumption in mechatronic systems is currently increasing because of the growing social awareness to achieve an environmentally sustainable future. Due to the current technology limitations, there is a significant loss of energy during the manufacturing process. Reducing these losses can be accomplished by developing new energy-efficient equipments [1] - [2]. However, energy consumption can also be reduced by developing novel energy optimal control algorithm, which is the topic of this paper. Park demonstrates a method that optimizes point-to-point motion profile to achieve minimal input energy by adopting Pontryagin's minimum principle in continuous time [3]. Sergaki presents a method that achieves robot speed-

\footnotetext{
${ }^{*}$ Corresponding author.
}

control requirements, with simultaneous minimization of total electromechanical losses [4] and Kim investigates a method that an energy-optimal trajectory is derived by minimizing the total energy drawn from the batteries [5]. These last two methods only consider the double integrator systems. The basic idea behind all these energy-optimal methods is that the objective function in the formulated optimization problem is set equal to the total energy consumption of the system.

An interesting on-line method for optimal control of mechatronic systems is Model Predictive Control (MPC) approach [6]. MPC became very popular in the eighties and since the end of the nineties, research on the development of fast MPC solution methods (fast MPC in short) has intensified to extend its application area to faster systems such as mechatronic or motion systems. One fast MPC approach is called explicit MPC [7], in which an analytical expression for the MPC control law is calculated by solving an optimization problem off-line. The method is efficient but limited to small-state dimensions problem. Another fast MPC approach is using fast numerical solution methods such as qpOASES to solve the optimal control problem in real-time.

This paper presents the developed energy-optimal MPC (EOMPC) strategy. It is based on the time-optimal (TOMPC) approach [8]. EOMPC aims at performing energy-optimal pointto-point motions within a given motion time. EOMPC solves this optimal control problem in real-time taking into account system constraints. Because of the real-time solution strategy, setpoint and motion time requirements can be changed during the motion. Energy optimality is achieved by setting the object function of the EOMPC optimization problem equal to the system's energy losses. The key issue is to utilize the strategy of the prediction horizon to ensure that the motion time is exactly equal to the 
required motion time.

Application of EOMPC on a badminton robot illustrates the developed approach. The badminton robot has 3 degrees of freedom - a linear axis, a rotational axis and a hit axis. The linear axis is used to position the robot across the field using a linear motor and it is the main energy consumer. Hence energy-optimal motion is only considered for this linear axis.

In the following section, more details of TOMPC and EOMPC are presented. The application of EOMPC on the robot is discussed in the third section. In the fourth section, simulation results are shown and an experimental comparison of EOMPC with TOMPC is provided in the fifth section. The conclusions are drawn in the last section.

\section{ENERGY OPTIMAL MPC}

This section first introduces the basic ideas of TOMPC. Then the EOMPC approach, which is based on TOMPC, is presented.

\section{Time-optimal MPC}

Both TOMPC and EOMPC are MPC approaches. MPC is an advanced control technique that determines the control action by solving on-line, at every sampling time $l$, an open-loop optimal control problem, based on the current state of the system $\hat{\mathbf{x}}_{l}$. The optimization generates an input sequence for a specified time horizon $N$. However, only the first input $\hat{u}_{l}=u_{0}$ is applied to the system. In its simplest setting, the optimization problem to be solved at each time step $l$ is:

$$
\begin{gathered}
V_{A}^{*}=\min _{\substack{x_{0}, \ldots, x_{N} \\
u_{0}, \ldots, u_{N-1}}} \sum_{k=0}^{N-1}\left\|u_{k}-u_{r e f}\right\|_{R}^{2}+\left\|x_{k}-x_{r e f}\right\|_{Q}^{2} \\
\text { s.t. } \quad \mathbf{x}_{0}=\hat{\mathbf{x}}_{l} \\
\mathbf{x}_{k+1}=\mathbf{A} \mathbf{x}_{k}+\mathbf{B} u_{k}, k=0,1, \cdots, N-1 \\
\mathbf{H} x_{k}+\mathbf{G} u_{k} \geq e, k=0,1, \cdots, N-1 \\
x_{N}=x_{r e f}
\end{gathered}
$$

where $\left(x_{0}, \ldots, x_{N}, u_{0}, \ldots, u_{N-1}\right)$ are the optimization variables over time horizon $N$ and $\hat{\mathbf{x}}_{l}$ in Eq. (1b) is the system state at time $l$. The optimization is based on a system model Eq. (1c) and acknowledges bounds on inputs, outputs and internal states Eq. (1d). Constraints Eq. (1e) is a moving endpoint constraint and requires the system to be at rest at the desired setpoint at time $N$. This constraint hence guarantees the stability, assuming semi-definite positivity of the weights $Q$ and $R$. Vectors $x_{r e f}$ and $u_{r e f}$ express together with a feasible equilibrium point, i.e. they satisfy $\mathbf{H} x_{r e f}+\mathbf{G} u_{r e f} \geq e$ and $\mathbf{x}_{r e f}=\mathbf{A} \mathbf{x}_{r e f}+\mathbf{B} u_{r e f}$, which is together with an output function also the defining function of these values. The input is optimized considering the Euclidean norm of the system input and state, weighted with $R$ and $Q$ respectively. Figure 1 summarizes the idea of MPC.

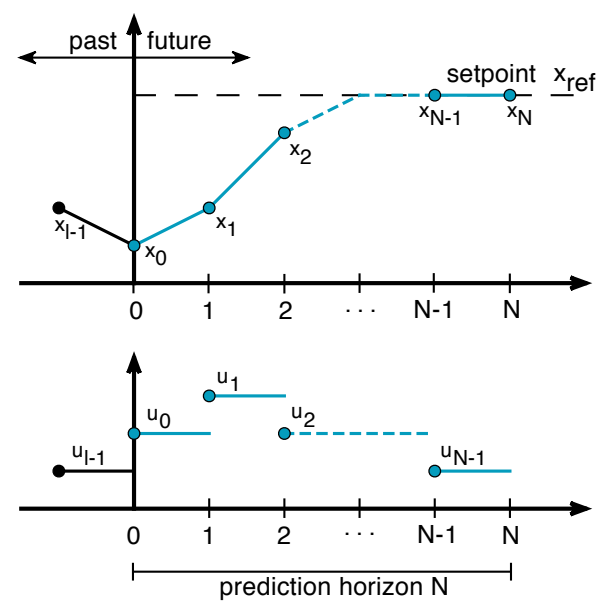

Figure 1. GENERAL IDEA OF MPC

TOMPC [8] is a MPC approach for time-optimal point-topoint motion control of linear time-invariant systems. It is formulated as a two layer optimization problem. First the settling time defined as the number $N$ of sampling instances required for the system to be at rest at the desired set point is minimized so that the time optimality is achieved. Once the minimal $N$ is reached, the optimal signal sequence is obtained by solving the MPC optimization problem. Hence, at each time step $l$ the following two optimization problems are to be solved:

1. Equation (1) is defined as 'Problem A' denoted by $P_{A}\left(\hat{\mathbf{x}}_{l}, N\right)$. Function $V_{A}^{*}=\infty$ if $P_{A}\left(\hat{\mathbf{x}}_{l}, N\right)$ is infeasible. From physics point of view, infeasibility of $P_{A}\left(\hat{\mathbf{x}}_{l}, N\right)$ means that the system can not be at rest at the desired setpoint $x_{\text {ref }}$ at time $N$ while respecting all constraints. Therefore, an admissible set $\mathbb{X}(N)$ is defined:

$$
\mathbb{X}(N)=\left\{\hat{\mathbf{x}}_{l} \mid P_{A}\left(\hat{\mathbf{x}}_{l}, N\right) \text { is feasible }\right\}
$$

from which the setpoint can be reached within $N$ time steps, while respecting all system constraints Eq. (1d).

2. 'Problem B' denoted by $P_{B}\left(\hat{\mathbf{x}}_{l}\right)$ is defined as follows:

$$
\begin{gathered}
V_{B}^{*}\left(\hat{\mathbf{x}}_{l}\right)=\min _{N \in \mathbb{N}} N \\
\text { s.t. } \quad \hat{\mathbf{x}}_{l} \in \mathbb{X}(N) \\
N_{\min } \leq N \leq N_{\max }
\end{gathered}
$$

where $N$ is bounded by $N_{\max }$ and $N_{\min } . N_{\max }$ is the maximal prediction horizon. To guarantee unconstrained solvability, $N_{\min }$ should be selected bigger than $n / n_{u}$ with $n$ the number of states and $n_{u}$ the number of inputs [9]. In practice $N_{\text {min }}$ is typically selected 2 to 3 times higher in order to obtain a less nervous control behaviour when the set point is reached [8]. If $\hat{\mathbf{x}}_{l} \in \mathbb{X}\left(N_{\min }\right)$, 
TOMPC optimization problem reduce to a regular MPC problem without endpoint constraints, which is a QP. If $\hat{\mathbf{x}}_{l} \notin \mathbb{X}\left(N_{\min }\right)$, the resulting optimization problem $P_{B}\left(\hat{\mathbf{x}}_{l}\right)$ is a mixed integer problem that can be solved by a series of feasibility problems $P_{A}\left(\hat{\mathbf{x}}_{l}, N\right)$.

In order to efficiently solve feasibility problem $P_{A}\left(\hat{\mathbf{x}}_{l}, N\right)$ using the qpOases online active set approach [10] - [11], it has to be reformulated into a QP of constant size. This is done by replacing the varying horizon length $N$ in Eq. (1a), Eq. (1c), and Eq. (1d), by its maximum value $N_{\max }$ and adding the following equality constraints that impose a constant control input once the reference state $x_{r e f}$ is reached:

$$
u_{k}=u_{r e f}, \quad k=N, \cdots, N_{\max }-1,
$$

qpOASES is an open-source $\mathrm{C}++$ implementation of the online active set strategy that is used to solve the TOMPC problem and the EOMPC problem.

\section{Blocking strategy}

A disadvantage of the TOMPC and EOMPC algorithm, is the dependence of the largest possible reference step on the value of $N_{\max }$ because endpoint constraints discussed below in Eq. (6f) and Eq. (6g) have to be satisfied for $N \leq N_{\max }$, that is, the system must be able to reach this largest possible reference position in no more than $N_{\max }$ time steps without violating the system constraints Eq. (6e). $N_{\max }$ itself is limited because it determines the size of the optimization problem and hence the worst case computation time of the solution method which is limited by the sampling period. In order to relieve this limitation, non-equidistant time steps or time gridding is applied such that larger time horizons can be considered without increasing the total number of discretization points $N_{\max }$ and hence the number of optimization variables. This strategy is called blocking, and typically for the first part of the horizon up to $N_{\min }$, the time step corresponds to the sampling period $T_{s}$. Thereafter, the time steps are gradually increased up to for example 10 times the sampling time. For the considered test case discussed in section 4, the applied blocking strategy yields the following discrete time instants with $N_{\max }=40$ :

$$
[\underbrace{1 \cdots 10}_{10} \underbrace{13 \cdots 40}_{10} \underbrace{47 \cdots 110}_{10} \underbrace{120 \cdots 210}_{10}] * T_{s}
$$

The time interval of the first, second, third, and fourth group of 10 time instants equal $T_{s}, 3 * T_{s}, 7 * T_{s}, 10 * T_{s}$ respectively. The resulting time horizon is $210 * T_{s}$, which is realized with only $N_{\max }=40$.

However a drawback of blocking is that it induces nonsmooth optimal solution. The non-smoothness is big in the beginning of the motion and then gets smaller when the setpoint is getting closer. This is because $N$, the number of sampling instants required for the system to be at rest at the desired setpoint is located in the end of the prediction horizon in the beginning of the motion and one change of $N$ causes a large time step in the endpoint constraints. During the motion, the remaining motion time decreases, hence $N$ moves forward and one change of $N$ causes a smaller time step in the endpoint constraints.

\section{Determination of the remaining motion time}

As mentioned above, energy-optimal point-to-point motion is considered for a given motion time $T . K^{*}$, which is used in the EOMPC algorithm discussed below in Eq. (6), is the index of the time instant in the prediction horizon at which the system has to be at rest at the desired endpoint position. $K^{*}$ depends of course on the desired motion time $T$ which is assumed to be a multiple of the sampling time $T_{s}$. Assume that a new setpoint and motion time $T=M * T_{s}$ are applied at $l=0$, and $l$ gradually increases at each sampling instant with one, then the remaining time available to reach the end point at time instant $l$ is $(M-l) * T s$. If no blocking is applied,the calculation of $K^{*}$ is straightforward that $K^{*}=M-l$. If blocking is applied, $K^{*}$ is found by looking for the earliest time instant $T_{K^{*}}$ in the non-equidistant prediction horizon that yields a time interval from the beginning of the prediction horizon up to that time instant, that is larger than or equal to $(M-l) * T s$. If distance between the time instant prior to $T_{K^{*}}$ in the prediction horizon is $W_{i} * T s$ seconds, $K^{*}$ will remain constant for $W_{i}$ consecutive sampling times after which it reduces with one.

\section{Energy-Optimal MPC}

EOMPC is a control method to realize energy optimal pointto-point motions within a required motion time. TOMPC can be developed to EOMPC if the objective function of problem $P_{A}\left(\hat{\mathbf{x}}_{l}, N\right)$ considers only energy losses, and the lower bound of $N_{\text {min }}$ in $P_{B}\left(\hat{\mathbf{x}}_{l}\right)$ is replaced by $\max \left(N_{\min }, K^{*}\right)^{1}$. Using only energy losses in the objective function and not a trade-off between input effort and tracking errors ensures that the motion becomes energy-optimal. By using the TOMPC approach to find the minimal motion time and limiting this time by the requested motion time, the motion will be executed in the given time. However, if this requested motion time is too short for the considered system and displacement considering the system constraints, the EOMPC approach, which will perform exactly the same as TOMPC in this case, will automatically find the shortest possible motion time and realize the motion in an time-optimal way. The EOMPC optimization problem is formulated similar as that of TOMPC. The two layer optimization problem of EOMPC consists of 'Problem E', $P_{E}\left(\hat{\mathbf{x}}_{l}, N\right)$, and 'Problem F', $P_{F}\left(\hat{\mathbf{x}}_{l}\right)$.

\footnotetext{
${ }^{1} \max (a, b)$ means the maximum of $a$ and $b$
} 


$$
\begin{aligned}
& V_{E}^{*}=\min _{\mathbf{u}} E_{\text {loss }}(\mathbf{u}) \\
& \text { s.t. } \quad \mathbf{x}_{0}=\hat{\mathbf{x}}_{l} \\
& \mathbf{x}_{k+1}=\mathbf{A}_{k} \mathbf{x}_{k}+\mathbf{B}_{k} u_{k}, \quad k=0,1, \cdots, N_{\max }-1 \quad k=0,1, \cdots, N_{\max } \\
& y_{k}=\mathbf{C} \mathbf{x}_{k}, \quad k=0, N_{\max }-1 \\
& \mathbf{H} x_{k}+\mathbf{G} u_{k} \geq e, \quad k=0,1, \cdots, N+n-1 \\
& y_{k}=y_{\text {ref }}, \quad k=N, \cdots, N+n, \quad k=N, \cdots, N_{\max }-1
\end{aligned}
$$

Equation (6) is defined as $P_{E}\left(\hat{\mathbf{x}}_{l}, N\right)$ and $k$ indicates the $k^{\text {th }}$ time instant of the considered prediction horizon, which consists of $N_{\max }$ non-equidistant time steps. $\mathbf{A}_{k}$ and $\mathbf{B}_{k}$ in Eq. (6c) are defined as following:

$$
\begin{gathered}
\mathbf{A}_{k}=\mathbf{A}^{i k} \\
\mathbf{B}_{k}=\left(\mathbf{A}^{i k-1}+\mathbf{A}^{i k-2}+\cdots+1\right) \mathbf{B}
\end{gathered}
$$

with $i k$ the number of sampling instants between the $k^{t h}$ and the $(k-1)^{t h}$ time instants. The admissible set $\mathbb{X}_{E}(N)$ is defined:

$$
\mathbb{X}_{E}(N)=\left\{\hat{\mathbf{x}}_{l} \mid P_{E}\left(\hat{\mathbf{x}}_{l}, N\right) \text { is feasible }\right\}
$$

and $P_{F}\left(\hat{\mathbf{x}}_{l}\right)$ is defined as follows:

$$
\begin{array}{r}
V_{F}^{*}\left(\hat{\mathbf{x}}_{l}, K^{*}\right)=\min _{N \in \mathbb{N}} N \\
\text { s.t. } \hat{\mathbf{x}}_{l} \in \mathbb{X}_{E}(N) \\
\max \left(N_{\min }, K^{*}\right) \leq N \leq N_{\max }
\end{array}
$$

Table 1 shows the algorithm of the EOMPC optimization problem. The input for this algorithm is the estimated current system state $\hat{\mathbf{x}}_{\mathbf{I}}$ and $K^{*}$. The following sections discuss the application of EOMPC on the linear axis of a badminton robot. Both numerical and experimental results are presented. The following section describes the badminton robot and formulates the EOMPC control problem for this test case.

\section{TEST CASE AND PROBLEM DESCRIPTION The considered setup}

The considered test setup is a badminton robot, the current mechanical implementation of which is shown in Fig. 2. The robot has 3 degrees of freedom - a linear axis, a rotational axis and a hit axis. The linear axis is used to position the robot across the field using a linear motor and it is the main energy consumer. The system is equipped with a stereo digital camera system to detect the shuttle cock. Based on shuttle cock location measurements, the shuttle cock trajectory is estimated. Interception logic
Table 1. ALGORITHM OF ENERGY OPTIMAL MPC

Algorithm 1 EOMPC optimization procedure

input: $\hat{\mathbf{x}}_{\mathbf{l}}, K^{*}$
output: $u^{*}$
start with initial guess for $N$
solve QP problem $P_{E}\left(\hat{\mathbf{x}}_{l}, N\right)$ Eq. $(6)$
if $P_{E}\left(\hat{\mathbf{x}}_{l}, N\right)$ feasible then
while $P_{E}\left(\hat{\mathbf{x}}_{l}, N\right)$ was feasible do
$\quad$ store $u^{*}=u_{0}\left(\hat{x}_{l}, N\right)$
$\quad N=N-1$
if $N \geq \max \left(N_{\text {min }}, K^{*}\right)$ then
$\quad$ solve QP problem $P_{E}\left(\hat{\mathbf{x}}_{l}, N\right)$
else
$\quad$ break
end if

end while

else

while $P_{E}\left(\hat{\mathbf{x}}_{l}, N\right)$ was infeasible do

$N=N+1$

if $N \leq N_{\max }$ then

solve QP problem $P_{E}\left(\hat{\mathbf{x}}_{l}, N\right)$

else

solve $P_{E}\left(\hat{\mathbf{x}}_{l}, N\right)$ without endpoint constraints

break

end if

end while

store $u^{*}=u_{0}\left(\hat{\mathbf{x}}_{l}, N\right)$

end if

determines the appropriate hit time and the robot configuration (i.e. hit angle, rotational angle and coordinate along the linear axis) in order to hit back the shuttle to the human opponent at a point along the predicted trajectory. Once the execution of the hitting happens, the robot moves back to the center of the linear motor where is set as the homing position with a given constant homing time and waits for the next desired setpoint requested.

It is assumed that the linear motor is a perfect velocity controlled system so that the system can be regarded as an integrator. The input and the output of the system are the velocity and the 


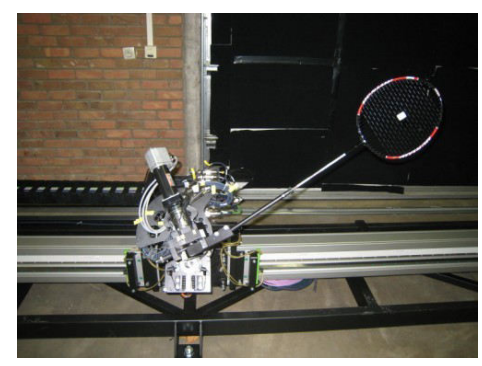

Figure 2. THE BADMINTON ROBOT

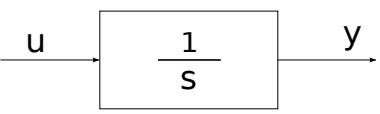

Figure 3. SYSTEM BLOCK DIAGRAM

position of the robot respectively as shown in Fig. 3. An additional variable $\delta \mathbf{u}$ is defined:

$$
\delta u_{k}=\frac{u_{k}-u_{k-1}}{\delta T_{k}}, \quad k=0,1, \cdots, N_{\max }-1
$$

where $u_{k}$ and $u_{k-1}$ are the control variables at $k^{t h}$ and $(k-1)^{t h}$ (non-equidistant) time instants in the prediction horizon, and $\delta T_{k}$ is the time difference between these two time instants. $\delta u_{k}$ corresponds to the system acceleration, and by introducing this additional variable, also constraints on the acceleration can be taken into account in the EOMPC formulation.

\section{Formulation of the optimization problem}

Assuming that friction forces of the linear motor are negligible, the motion energy losses, $E_{\text {loss }}$, are copper losses in the motor, which is proportional to square of the motor current and hence proportional to square of the acceleration of the motor, as shown in Eq. (11),

$$
E_{\text {loss }}=T_{s} * \sum_{k=0}^{N_{\max }-1} c * \delta u_{k}^{2}
$$

where $c$ is a constant depending on the ohmic resistance of the motor windings. Due to the limitations of the system, such as peak current limitations and the total length of the linear motor, the limits on the position, the velocity, and the acceleration, which are $\pm 1.9[\mathrm{~m}], \pm 3[\mathrm{~m} / \mathrm{s}], \pm 30\left[\mathrm{~m} / \mathrm{s}^{2}\right]$ respectively, are taken into account.

$$
P_{E}\left(\hat{\mathbf{x}}_{l}, N\right) \text { of the EOMPC optimization problem is defined as }
$$
following:

$$
\begin{gathered}
\min _{\dot{\mathbf{u}}} \dot{\mathbf{u}}^{\mathrm{T}} \mathbf{R} \dot{\mathbf{u}} \\
\text { s.t. } \quad \mathbf{x}_{0}=\hat{\mathbf{x}}_{l} \\
\mathbf{x}_{k+1}=\mathbf{A}_{k} \mathbf{x}_{k}+\mathbf{B}_{k} u_{k}, \quad k=0,1, \cdots, N_{\max }-1 \\
y_{k}=\mathbf{C} \mathbf{x}_{k}, \quad k=0,1, \cdots, N_{\max } \\
\delta u_{k}=\frac{u_{k}-u_{k-1}}{\delta T_{k}}, \quad k=0,1, \cdots, N_{\max }-1 \\
\mathbf{H} x_{k}+\mathbf{G} u_{k} \geq e, \quad k=0,1, \cdots, N_{\max }-1 \\
\delta u_{\min }, u_{\min }, y_{\min } \leq \delta u_{k}, u_{k}, y_{k} \leq \delta u_{\max }, u_{\max }, y_{\max } \\
y_{k}=y_{\text {ref }}, \quad k=N, \cdots, N+n-1 \\
u_{k}=u_{\text {ref }}, \quad k=N, \cdots, N_{\max }-1
\end{gathered}
$$

where $R$ is a positive definite matrices. This optimization problem is a convex QP because the dynamics of the system and the constraints are linear. As a result of the constant size of the optimization problem during the feasibility search, the on-line active set method qpOASES can be utilized to solve this optimization problem.

\section{SIMULATION RESULTS}

The following simulation is implemented: a desired setpoint $Y_{\text {ref }}=2[\mathrm{~m}]$ is required at time instant $0.1[\mathrm{~s}]$ with the required motion time $T=1[\mathrm{~s}]$, the sampling frequency $f_{s}=200[\mathrm{~Hz}]$. The results of the simulation are shown in Fig. 4.

Figure 4 (top) shows that the system moves from $0[\mathrm{~m}]$ to $2[m]$ during the time instant from $0.1[s]$ to $1.1[s]$. The real motion time is exactly equal to the required motion time $T$. Figure 4 (middle) shows the velocity of the system that is zero in the beginning until there's a new request of desired setpoint $Y_{r e f}=2$ at $0.1[s]$. From $0.1[s]$, the velocity starts to increase until it reaches the upper bound value $3[\mathrm{~m} / \mathrm{s}]$ and keeps the maximum value for a short while, then it starts to decrease and reach $0[\mathrm{~m} / \mathrm{s}]$ at $1.1[\mathrm{~s}]$. This indicates that the system stays still at the desired setpoint at 1.1[s]. The acceleration of the system shown in Fig. 4 (bottom) increases immediately to the maximal value which is required to achieve energy-optimal point-to-point motion and then linearly decreases to the minimal value. This acceleration behaviour has been illustrated the energy-optimal solution in [12]. The fact that the non-smoothness of the acceleration is large in the beginning of the motion and then gets smaller is due to the blocking.

\section{EXPERIMENTAL VALIDATION}

In the experimental validation, with the sampling rates up to $100 \mathrm{~Hz}$, EOMPC is utilized as a feed-forward controller to generate a velocity trajectory so that the robot performs energyoptimal point-to-point motion as shown in Fig. 5 and the experimental validation results of the EOMPC are shown in Fig. 6. In order to compare the different behaviour of the system using the 

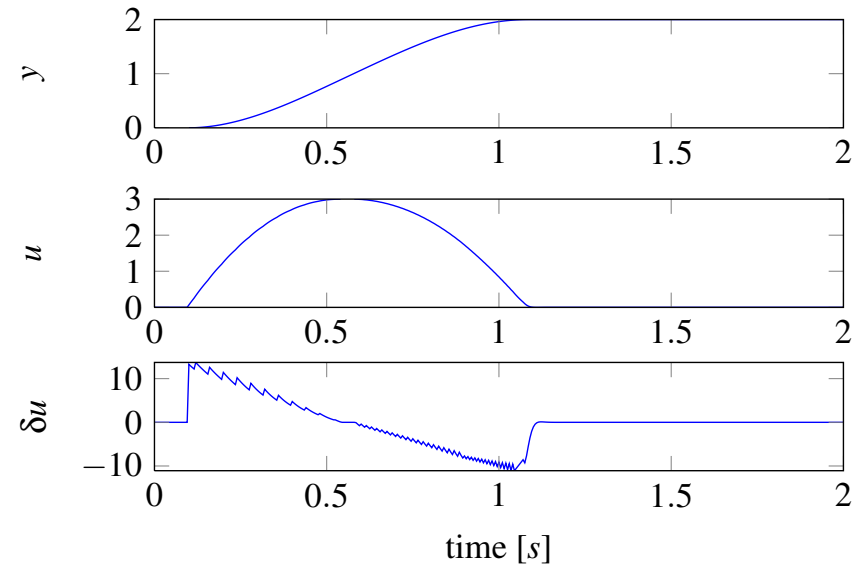

Figure 4. POSITION $(y)$, VELOCITY $(u)$, AND ACCELERATION $(\delta u)$ OF THE SYSTEM

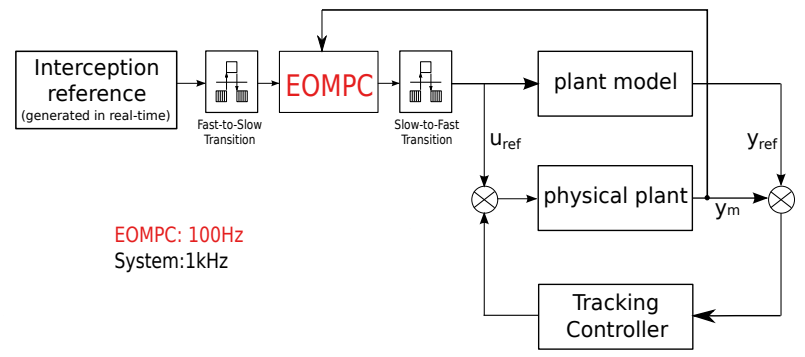

Figure 5. CONTROL ARCHITECTURE OF THE SYSTEM

EOMPC and the TOMPC, the experimental validation results of the TOMPC are also shown in Fig. 6 (b).

The requested motion time $T$ for several different motions is shown in Fig. 6(a). When a new displacement is requested as shown in Fig. 6(b) (dashed line), the motion time jumps to the given value and after that gradually decreases until it reaches zero, at which time the position of the system using the EOMPC arrives at the requested setpoint as shown in Fig. 6(b) (solid line) and hence the hitting of the shuttle is executed. Once the execution of the hitting happens, the system moves back to it's homing position 'zero' with a fixed homing time which is 0.5 seconds in this case and waits for the next desired setpoint requested. Fig. 6(c) and Fig. 6(d) show the velocity and the acceleration of the system using the EOMPC, both of which satisfy the system constraints.

Figure 6(b) compares the performance of the EOMPC (solid line) and the TOMPC (dash-dotted line) approach. When a new displacement is requested, the position of the system using the TOMPC arrives at the requested setpoint as fast as possible regardless of the required motion time as shown in Fig. 6(b). By using EOMPC, the system moves as fast as needed. The comparison of these two results shows the energy losses of the EOMPC is $39.62 \%$ less than that of the TOMPC that demonstrates energy (a)

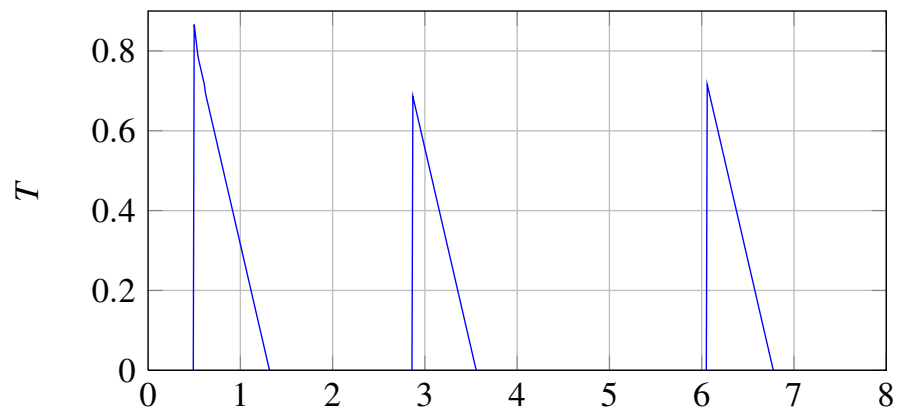

(b)

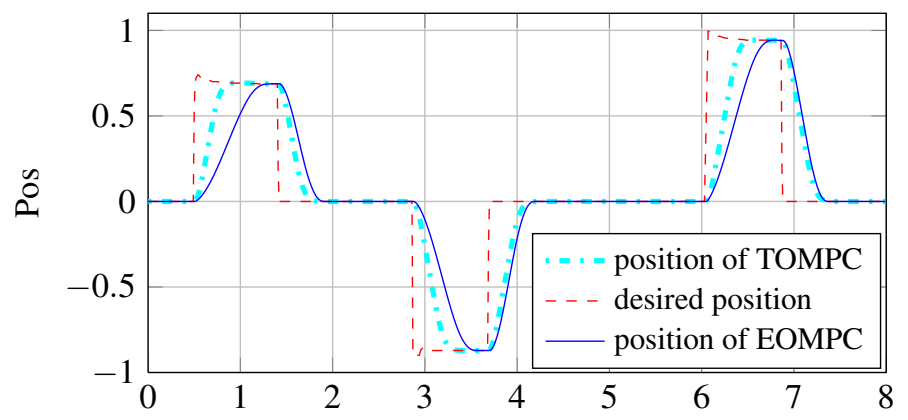

(c)

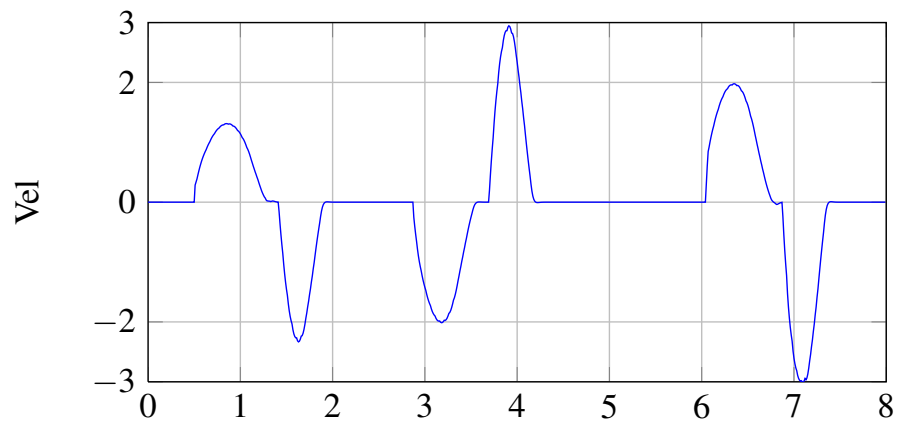

(d)

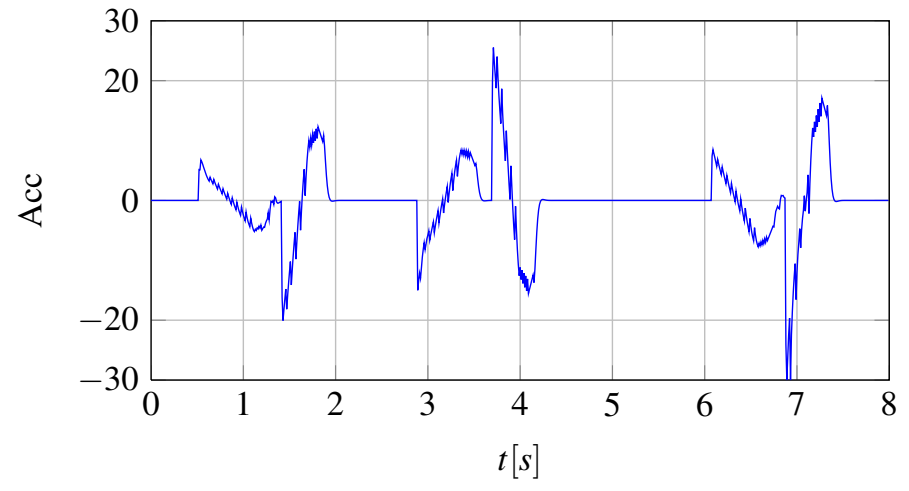

Figure 6. REQUIRED MOTION TIME $T$ (a), DESIRED POSITION , POSITION OF EOMPC, POSITION OF TOMPC (b), VELOCITY OF EOMPC (c) AND ACCELERATION OF EOMPC (d) OF THE EXPERIMENTAL VALIDATION 
efficiency is achieved with EOMPC.

\section{CONCLUSION}

This paper presents and experimentally validates energyoptimal MPC (EOMPC) aiming at performing energy optimal point-to-point motions. The energy optimal solution can be obtained by solving the EOMPC optimization problem. The key problem is to ensure the motion time requirement satisfied by using blocking strategy, which achieves sufficiently long prediction time to allow large displacements with a limited number of control variables. Then based on both the blocking and the motion time, the index of the time instant is determined to ensure the system arrives at the interception point within the required motion time. The experimental validation of EOMPC on the the badminton robot shows that the EOMPC is applicable for the mechatronic systems performing energy-optimal point-to-point motions within a required motion time. The comparison of the EOMPC and the TOMPC illustrates that the energy efficiency is achieved by this energy optimal MPC method.

\section{ACKNOWLEDGMENT}

This work has been carried out within the framework of projects IWT-SBO 80032 (LeCoPro) of the Institute for the Promotion of Innovation through Science and Technology in Flanders (IWT-Vlaanderen). This work also benefits from K.U.Leuven-BOF PFV/10/002 Center-of-Excellence Optimization in Engineering (OPTEC), the Belgian Programme on Interuniversity Attraction Poles, initiated by the Belgian Federal Science Policy Office (DYSCO), and K.U.Leuvens Concerted Research Action GOA/10/11.

\section{REFERENCES}

[1] Famouri, P., and Cooley, W., 1994. "Design of dc traction motor drives for high efficiency under accelerating conditions". IEEE Transactions on Industry Applications, 30(4), pp. 1134-1138.

[2] Cathey, J., 1979. "Reduction of dc traction motor armature copper losses through optimal control". Electric Machines and Power Systems, 4, pp. 269-283.

[3] J.S.Park, 1996. "Motion profile planning of repetitive point-to-point control for maximum energy conversion efficiency under acceleration conditions". Mechatronics, 6(6), p. 649663.

[4] Sergaki, E. S., Stavrakakis, G. S., and Pouliezos, A. D., 2002. "Optimal robot speed trajectory by minimization of the actuator motor electromechanical losses". Journal of Intelligent and Robotic Systems, 33(2), pp. 187-207.

[5] Kim, C. H., and Kim, B. K., 2007. "Minimum-energy translational trajectory generation for differential-driven wheeled mobile robots". Journal of Intelligent and Robotic Systems, 49(4), pp. 367-383.
[6] Morari, M., Lee, J. H., and Garca, C. E., 2002. "Model predictive control". pp. 27-33.

[7] Maurovic, I., Baotic, M., and Petrovic, I., 2011. "Explicit model predictive control for trajectory tracking with mobile robots". IEEE Conference on Advanced Intelligent Mechatronics (AIM), pp. 712-717.

[8] Van den Broeck, L., Diehl, M., and Swevers, J., 2011. “A model predictive control approach for time optimal pointto-point motion control". Mechatronics, 21(7), pp. 12031212.

[9] Kailath, T., 1980. "Linear systems". Prentice Hall.

[10] Ferreau, H., H.G., B., and M., D., 2008. "An online active set strategy to overcome the limitations of explicit mpc". International Journal of Robust and Nonlinear Control,

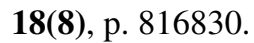

[11] Ferreau, H., 2006. "An online active set strategy for fast solution of parametric quadratic programs with applications to predictive engine control".

[12] Kluchev, V., 2001. Electric drive theory. Enegroatomizdat. 